\title{
Improved Semi Analytical Model of Effective Area for a Step and Parabolic-Index Optical Fiber: Introduction of Increased Parameters in Fundamental Modal Field Solution
}

\author{
Raja Roy Choudhury \\ Associate professor, Applied \\ Electronics and Instrumentation \\ Department, \\ Sikkim Manipal Institute of \\ Technology, \\ Majitar-737136. Sikkim, INDIA
}

\author{
Arundhati Roy Choudhury \\ Associate professor, \\ Physics Department, \\ Sikkim Manipal Institute of \\ Technology, \\ Majitar-737136. Sikkim, INDIA
}

\author{
Mrinal Kanti Ghose \\ Professor \\ Computer Science Department. \\ Sikkim Manipal Institute of \\ Technology, \\ Majitar-737136. Sikkim, INDIA,
}

\begin{abstract}
An accurate three parameter approximate solution of fundamental mode, to describe different propagation parameter of graded-index optical fiber has been presented. Due to three parameter optimization of the fundamental modal solution, the results are much more accurate for a wide range of optical fiber specifications. Employing variational technique, an estimation of effective area and effective index with proposed fundamental modal field are carried out. It has been shown that the results match identically with the exact available results for step and parabolic refractive index profile optical fiber. The accurate analytical expressions for the evaluation of effective area and effective index are formulated. Optimization of three parameters has been carried out for a wide range of normalized frequencies. Data for these parameters are also provided, which can be used directly while calculating different design parameters of an optical fiber.
\end{abstract}

\section{Key Words}

Single mode fiber, Effective Area, Nelder-Mead.

\section{INTRODUCTION}

Optical fibers in telecommunication systems now carry more channels and higher optical powers than ever before. Systems are operating in which the fiber carries such a high optical power density that signals can modify the transmission properties of the fiber. Hence, an accurate choice of fundamental modal field leads to exact description of propagation characteristics of an optical fiber. Initially, Gaussian approximation [1] was used to describe fundamental field, to provide simple analytical expressions for modeling an optical fiber. But, Gaussian expression fails to give accurate result for lower values of normalized frequency. Also, it is not useful to estimate evanescent field exactly. Many attempts have been made to achieve higher accuracy than the Gaussian field, such as, Gaussian exponential expression [2], Gaussian-Hankel [3], the extended Gaussian [4], the generalized Gaussian [5] and the Laguerre-Gauss/Bessel expansion approximation [6]. These modified approximations are much more accurate than the Gaussian field. Among these, Gaussian exponential expression[2] provides good result near the cut off frequency of next higher mode, but does not give accurate result for lower range of normalized frequencies[3]. An analytical expression without any requirement of optimization has also been reported [7]. But, such an expression may not be capable to meet all specifications of an optical fiber. Moreover, efficient optimizing software package is readily available in the market. So, dynamic optimization for all specification of the optical fiber can be preferred as an appropriate process, capable to satisfy variety of designer's demands.

All approximations for fundamental modal field reported so far, are limited to one or two variational parameters. But, recent advent of fiber manufacturing technology is associated with various design specifications. Thus, to accommodate all kinds of design and more flexibility in terms of three parameter approximation in formulating various propagation parameters of graded-index fibers has been introduced. Implementing variational technique, which is considered to be the most appropriate technique [8] for such kind of calculations, it has been demonstrated that the proposed approximation provides much more accurate analytical expressions to characterize variety of fiber behaviors. Again, the optimization process requires accurate analytical expression for propagation constant $\beta$, and core parameter $U$, which involve various fiber parameters, such as, core radius $(a)$, wavelength $(\lambda=2 \pi / k)$, refractive indices of core and cladding $\left(n_{\mathrm{co}}\right.$ and $\left.n_{\mathrm{cl}}\right)$. Hence, any desired specification can be substantiated by varying these fiber parameters.

Nonlinear effects occur more efficiently in optical fibers than in bulk samples of their constituent materials because the optical field is confined to the small fiber core area over long distances. The confinement of the optical field within the core is achieved by the refractive index profile, which determines the field distribution of the fundamental mode. In general, optical power density is given by the optical power divided by the area over which it is distributed. The field of the fundamental mode of a single mode fiber bears little resemblance to the refractive index profile and therefore the area of the doped core region itself does not truly represent the area of the mode field. The effective area, $A_{\text {eff }}$, of the mode must be calculated from the field distribution but since this falls gradually to zero away from the fiber axis, some operational definition of effective area is required. A similar problem arises with respect to defining the mode field diameter (MFD) of single mode fibers. The proposed semi analytical techniques can be used to measure the mode field distribution of a single mode fiber and can therefore be used to calculate the effective area.

This report presents to highlight that the proposed approximation is capable to estimate the fiber effective area over a wide range of normalized frequency, the parameters which are being evaluated are, effective index, effective area, of a step and parabolic-index optical fiber, with the proposed formulation of fundamental field. All results from calculations are in excellent agreement with the exact result. 
In the proposed semi analytical formulation, Nelder-Mead method $[9,10,12]$ of nonlinear unconstrained minimization have been used due to the fact that it is a direct search method [11] i.e. it does not require any derivative information, so it can optimize non-stationary functions and thus it can optimize core parameter U more efficiently[9, 10, 12]. Due to large scale availability of optimizing software in present day's market which enables dynamic optimization of core parameter $U$ for each and every specification of optical fiber possible. This renders the use of optimization of core parameter $U$ to be an efficient and computationally appropriate process. The NelderMead simplex method gained popularity very quickly. At earlier time, due to its simplicity and low storage requirements, it was ideally suited for use on minicomputers, especially in laboratories i.e. computation burden is less for this method [13]. Despite its age and recent advances in direct search methods, the Nelder-Mead method is still among the most popular direct search methods in practice[11].Therefore, it can be stated that our three parameter fundamental modal field solution coupled with Nelder-Mead Simplex method for nonlinear unconstrained minimization yields computationally appropriate results $[14$, $15]$.

\section{THEORY}

\subsection{Basic formulations}

For a weakly guiding fiber, the refractive index profile is given by,

$$
\begin{array}{ll}
n^{2}(R)=n_{2}^{2}+\left(n_{1}^{2}-n_{2}^{2}\right) f_{1}=n_{f_{1}} & \text { for, } R \leq 1 \\
n^{2}(R)=n_{2}{ }^{2}+\left(n_{1}^{2}-n_{2}^{2}\right) f_{2}=n_{f 2} & \text { for, } \quad R>1
\end{array}
$$

Here the normalized profile function is given by,

$$
\begin{array}{ll}
f_{1}=1 & \text { for, step-index profile, } \\
f_{1}=1-\mathrm{R}^{2} & \text { for, parabolic-index profile, }
\end{array}
$$

and, $f_{2}=0$

Here, $R(=r / a)$ is the normalized radius, $a$ is the core radius, $n_{1}$ and $n_{2}$ being the refractive indices of core axis and cladding respectively, $r$ is the actual radius of the optical fiber.

The proposed three parameter approximation for the fundamental mode as the trial field is as follows,

$\psi=\left[1+\left\{\alpha\left(R / R_{0}\right)^{2}\right\} / \mu\right]^{-(\mu+1) / 2}$

To employ variational technique, first we consider the expression of propagation constant $\beta$,

$\beta=\frac{1}{\left\langle\psi^{2}\right\rangle}\left[\int_{0}^{\infty} k^{2} n^{2}(R)|\psi|^{2} R d R-\frac{1}{a^{2}}\left\langle\psi^{\prime 2}\right\rangle\right]$

where, $\quad\left\langle\psi^{2}\right\rangle=\int_{0}^{\infty}|\psi|^{2} R d R$

and, $\left\langle\psi^{\prime 2}\right\rangle=\int_{0}^{\infty}\left|\frac{d \psi}{d R}\right|^{2} R d R$

Applying equation (1), we obtain,

$$
\beta=\frac{1}{\left\langle\psi^{2}\right\rangle}\left[\int_{0}^{1} k^{2} n_{f_{1}}|\psi|^{2} R d R+\int_{1}^{\infty} k^{2} n_{f_{2}}|\psi|^{2} R d R-\frac{1}{a^{2}}\left\langle\psi^{\prime 2}\right\rangle\right]
$$

Here, $k$ is the free space wave number. Now, the core parameter $U$ is given by,

$U^{2}=a^{2}\left(k^{2} n^{2}-\beta^{2}\right)$
Here $\mathrm{n}$ is the refractive index of the core axis and a is the core radius. For a fixed value of normalized frequency $V, U$ is minimized with respect to the variational parameters $\alpha, R_{0}$ and $\mu$. With the optimized values of these parameters, propagation constant $\beta$ and other characteristic parameters of an optical fiber can be evaluated.

\subsubsection{Effective Index $\left(n_{\text {eff }}\right)$ :}

The Effective index of an optical fiber is defined as,

$n_{\text {eff }}=\frac{\beta}{k}$

Once propagation constant $\beta$ is found, effective index can be obtained from the above equation.

2.1.2. Effective Area $\left(A_{e f f}\right)$ :

The effective area of an optical fiber is defined as,

$$
A_{\text {eff }}=2 \pi a^{2} \frac{\left\langle\psi^{2}\right\rangle}{\int_{0}^{\infty} \psi^{4}(R) R d R}
$$

Integrals given in (8) can be evaluated analytically by using the expression of fundamental modal field given in (2). Hence, effective area can be formulated.

\subsection{Evaluation of integrals:}

$$
\text { Let, } e_{1}=\left\{\left(10^{4} R_{0}^{2} \mu+\alpha\right) /\left(R_{0}^{2} \mu\right)\right\}^{\mu}, e_{2}=\{(\mu+\alpha) / \mu\}^{\mu} \text {, }
$$

$$
\begin{aligned}
& e_{3}=\left\{\left(R_{0}^{2} \mu+10^{6} \alpha\right) /\left(R_{0}^{2} \mu\right)\right\}^{\mu}, \\
& e_{4}=\left\{\left(R_{0}^{2} \mu+\alpha\right) /\left(R_{0}^{2} \mu\right)\right\}^{\mu}, \\
& e_{5}=10^{4 \mu} e_{2}, e_{6}=\left\{\left(R_{0}^{2} \mu+25 \alpha\right) /\left(R_{0}^{2} \mu\right)\right\}^{\mu}, \\
& \quad(11.1) \\
& e_{7}=\left(10^{4} R_{0}^{2} \mu+\alpha\right)^{2} \text { and } e_{8}=\left(R_{0}^{2} \mu+25 \alpha\right)^{2} .
\end{aligned}
$$

\subsubsection{Analytical expression for propagation} constant $(\beta)$ :

$$
\left\langle\psi^{2}\right\rangle=\frac{1}{2} R_{0}^{2}\left(\frac{\left(-e_{1}+10^{4 \mu} e_{2}\right)}{\alpha e_{1} e_{2}}-\frac{\left(e_{2}+e_{3}\right)}{\alpha e_{2} e_{3}}\right)
$$




$$
\begin{aligned}
& \left\langle\psi^{\prime^{2}}\right\rangle=\left[\mu /\left\{2 a^{2}(\mu+2) e_{2}(\mu+\alpha)^{2}\right\}\right]\left[e _ { 1 } \left(\alpha^{3} \mu^{2}+\alpha^{2} \mu^{2}+2 \alpha^{2} \mu^{2}+\right.\right. \\
& \left.2 \alpha^{3}+\alpha^{2} \mu+3 \alpha^{3} \mu\right)+10^{8} R_{0}^{4} e_{1}\left(3 \mu^{4}+3 \alpha \mu^{3}+2 \alpha \mu^{2}+\alpha \mu^{4}+\mu^{3}\right) \\
& +2 \times 10^{4} R_{0}{ }^{2} e_{1}\left(\alpha^{2} \mu^{3}-6 \alpha^{3} \mu^{2}+2 \alpha^{2} \mu+\alpha \mu^{3}+\alpha \mu^{2}+3 \alpha^{2} \mu^{2}\right) \\
& -e_{5} 10^{4} R_{0}^{2}\left(6 \alpha^{2} \mu^{2}-3 R_{0}^{2} \alpha^{3} \mu-2 \alpha \mu^{2}-4 \alpha^{2} \mu-2 \alpha^{2} \mu^{3}-2 \alpha^{3}\right. \\
& \left.-3 \alpha \mu^{3}-\alpha \mu^{4}\right)-10^{8} R_{0}^{4} e_{5}\left(\mu^{4}+2 \alpha \mu^{2}+\mu^{3}+\alpha^{2} \mu+2 \alpha^{2} \mu^{2}\right) \\
& \left.\left.-10^{4} R_{0}^{2} e_{2} \alpha^{3} \mu^{2}\right\} / e_{1} e_{7}\right]+\left[e _ { 3 } \left\{10^{12}\left(\mu^{2} \alpha^{2}+\mu^{2} \alpha^{3}+3 \mu \alpha^{3}+\mu \alpha^{2}+2 \alpha^{3}\right)\right.\right. \\
& +\mu^{4} R_{0}{ }^{4}+2 \times 10^{6} R_{0}{ }^{2}\left(\mu^{2} \alpha+3 \mu^{2} \alpha^{2}+2 \mu \alpha^{2}+R_{0}{ }^{2} \mu^{3} \alpha+\mu^{3} \alpha^{2}\right)+ \\
& \left.R_{0}^{4}\left(2 \mu^{2} \alpha+\mu^{4} \alpha+\mu^{3}+3 \mu^{3} \alpha\right)\right\}-e_{2}\left\{1 0 ^ { 6 } R _ { 0 } { } ^ { 2 } \left(2 \alpha^{3}+\mu^{4} \alpha+\right.\right. \\
& \left.\mu^{2} \alpha^{3}+2 \mu^{3} \alpha^{2}+3 \mu^{3} \alpha+3 \mu \alpha^{3}+2 \mu^{2} \alpha+4 \mu \alpha^{2}+6 \mu^{2} \alpha^{2}\right)+ \\
& \left.\left.\left.R_{0}^{4}\left(\mu \alpha^{2}+2 \mu^{2} \alpha+2 \mu^{3} \alpha+\mu^{3}+\mu^{2} \alpha^{2}+\mu^{4}\right)\right\}\right] /\left\{e_{3}\left(R_{0}^{2} \mu+10^{6} \alpha\right)^{2}\right\}\right]
\end{aligned}
$$

$\int_{0}^{1} k^{2} n_{f_{1}}|\psi|^{2} R d R=\left\{R_{0}^{2} k^{2} n_{1}^{2} /\left(2 \alpha e_{2}\right)\right\}\left[\left\{\left(e_{5}-e_{1}\right) / e_{1}\right\}-\left\{\left(e_{2}-e_{4}\right) / e_{4}\right\}\right]$

$$
\begin{aligned}
& \int_{0}^{1} k^{2} n_{f_{1}}|\psi|^{2} R d R=\left[\left\{5 \times 10^{-5} R_{0}{ }^{2} k^{2}\right\} / e_{1} e_{4} \alpha^{2}\right. \\
& (\mu-1)]\left[10^{4} e_{1}\left(\alpha\left(n_{1}{ }^{2}-\mu n_{2}{ }^{2}\right)+R_{0}{ }^{2} \mu\left(n_{1}{ }^{2}-n_{2}{ }^{2}\right)\right)\right. \\
& \left.+10^{4 \mu} e_{4}\left\{\alpha \mu\left(n_{2}{ }^{2}+9999 n_{1}{ }^{2}\right)-10^{4}\left(\alpha n_{1}{ }^{2}+R_{0}{ }^{2} \mu\left(n_{1}{ }^{2}-n_{2}{ }^{2}\right)\right)\right\}\right]
\end{aligned}
$$

for, parabolic-index profile

$\int_{1}^{\infty} k^{2} n_{f_{2}}|\psi|^{2} R d R=-R_{0}{ }^{2} k^{2} n_{2}{ }^{2}\left\{\left(e_{4}-e_{3}\right) /\left(2 \alpha e_{3} e_{4}\right)\right\}$

$$
\int_{1}^{R_{0}} k^{2} n_{f_{2}}|\psi|^{2} R d R=\mathrm{R}_{0}{ }^{2} k^{2} n_{2}{ }^{2}\left(\mathrm{e}_{2}-\mathrm{e}_{4}\right) /\left(2 \alpha \mathrm{e}_{2} \mathrm{e}_{4}\right)
$$

$$
\int_{R_{0}}^{\infty} k^{2} n_{f_{2}}|\psi|^{2} R d R=-R_{0}^{2} k^{2} n_{2}^{2}\left\{\left(e_{2}-e_{3}\right) /\left(2 \alpha e_{2} e_{3}\right)\right\}
$$

$$
\text { (14e) }
$$

Putting (12) - (14) in (5), we get analytical expression for propagation constant $\beta$, and hence, expression for $U$ can be obtained from (6)

\subsubsection{Analytical expressions for Effective Index} $\left(n_{\text {eff }}\right)$ :

Once propagation constant $\beta$ is obtained, effective index can be estimated from (7).

\subsubsection{Analytical expressions for Effective Area $\left(A_{\text {eff }}\right)$ :}

$$
\begin{aligned}
& \int_{0}^{\infty} \psi^{4}(R) R d R=\left[R_{0}^{2} \mu^{2} /\left\{2 e_{2}^{2} \alpha(\mu+\alpha)(2 \mu+1)\left(10^{4} R_{0}^{2} \mu+\alpha\right)\right\}\right. \\
& ]\left[\left\{10^{4+8 \mu} e_{2}^{2} R_{0}^{2}(\mu+\alpha)-e_{1}^{2}\left(10^{4} R_{0}^{2} \mu-\alpha\right)\right\} / e_{1}^{2}\right. \\
& \left.-\left\{R_{0}^{2} e_{2}^{2}(\mu+\alpha)-e_{3}^{2}\left(R_{0}^{2} \mu+10^{6} \alpha\right)\right\} / e_{3}^{2}\right]
\end{aligned}
$$

Substituting (12) and (15), in equation (8), analytic expression of effective area $\left(A_{e f f}\right)$ can be formulated.

\subsection{Nelder-Mead Simplex Method for Nonlinear Unconstrained Optimization [12]}

The general Nelder-Mead Simplex Method can be given as:

1) Construct the initial working simplex $S$

2) Repeat the following steps until the termination test is satisfied:

a) calculate the termination test information;

b) If the termination test is not satisfied then transform the working simplex.

3) Return the best vertex of the current simplex $S$ and the associated function value.

Even though the method is quite simple, it can be implemented in many different ways. Apart from some minor computational details in the basic algorithm, the main difference between various implementations lies in the construction of the initial simplex, and in the selection of convergence or termination tests used to end the iteration process, as stated hereunder.

\subsubsection{Initial simplex}

The initial simplex $S$ is usually constructed by generating $(n+1)$ vertices $x_{0}, x_{1}, \ldots \ldots x_{n}$ around a given input point $x_{i n} \in \mathbf{R}^{n}$. In practice, the most frequent choice is $x_{0}=x_{i n}$ to allow proper restarts of the algorithm. The remaining $n$ vertices are then generated to obtain one of two standard shapes of $S$ :

- $\quad S$ is right-angled at $\mathrm{x}_{0}$, based on coordinate axes, or $x_{j}:=x_{0}+h_{i} e_{j}, \quad j=1, \ldots \ldots, n$, where $h_{j}$ is a step size in the direction of unit vector $\boldsymbol{e}_{j}$ in $\mathbf{R}^{n}$

- $\quad S$ is a regular simplex, where all edges have the same specified length.

\subsubsection{Simplex transformation algorithm}

One iteration of the Nelder-Mead method consists of the following three steps.

1. Ordering: Determine the indices $h, s, l$ of the worst, second worst and the best vertex, respectively, in the current working simplex $S$ 
$f_{h}=\max _{j} f_{j}, \quad f_{s}=\max _{j \neq h} f_{j}, \quad f_{l}=\min _{j \neq h} f_{j}$.

In some implementations, the vertices of $S$ are ordered with respect to the function values, to

satisfy $f_{0} \leq f_{1} \leq \ldots \ldots . . \leq f_{n-1} \leq f_{n}$. Then

$l=0, s=n-1$, and $h=n$. Consistent tie-breaking rules for this ordering were given by [9].

2. Centroid: Calculate the centroid $\boldsymbol{c}$ of the best sidethis is the one opposite the worst vertex $x_{h}$

$$
c:=\frac{1}{n} \sum_{j \neq h} x_{j} \text {. }
$$

3. Transformation: Compute the new working simplex from the current one.

- $\quad$ First, try to replace only the worst vertex $x_{h}$ with a better point by using reflection, expansion or contraction with respect to the best side.

- $\quad$ All test points lie on the line defined by $x_{h}$ and $c$, and at most two of them are computed in one iteration.

- If this succeeds, the accepted point becomes the new vertex of the working simplex.

- If this fails, shrink the simplex towards the best vertex $x_{l}$. In this case, $n$ new vertices are computed.

Simplex transformations in the Nelder-Mead method are controlled by four parameters: $\alpha$ for reflection, $\beta$ for contraction, $\gamma$ for expansion and $\delta$ for shrinkage. These should satisfy the following constraints

$\alpha>0,0<\beta<1, \gamma>1, \gamma>\alpha, 0<\delta<1$.

The standard values, used in most implementations, are $\alpha=1, \beta=\frac{1}{2}, \gamma=2, \delta=\frac{1}{2}$.

A slightly different choice was suggested by Parkinson and Hutchinson [10]. An alternate notation for these four parameters: $\rho, \gamma, \chi, \sigma$, respectively, is used in [9] and Matlab implementation.

The following algorithm describes the working of simplex transformations in step 3 [11], and the effects of various transformations are shown in the corresponding figures.

Reflect: Compute the reflection point

$x_{r}:=c+\alpha\left(c-x_{h}\right)$ and $f_{r}:=f\left(x_{r}\right)$. If

$f_{l} \leq f_{r}<f_{s}$, accept $x_{r}$ and terminate the iteration

- Expand: If $f_{r}<f_{l}$, compute the expansion point $x_{e}:=c+\gamma\left(x_{r}-c\right)$ and $f_{e}:=f\left(x_{e}\right)$. If $f_{e}<f_{r}$, accept $x_{e}$ and terminate the iteration. Otherwise (if $f_{e} \geq f_{r}$ ), accept $x_{r}$ and terminate the iteration.

This "greedy minimization" approach includes the better of the two points $x_{r}, x_{e}$ in the new simplex, and the simplex is expanded only if $f_{e}<f_{r}<f_{l}$. It is used in most implementations, and in theory [9]. The original Nelder-Mead paper [12] uses "greedy expansion", where $x_{e}$ is accepted if $f_{e}<f_{l}$ and $f_{r}<f_{l}$, regardless of the relationship between $f_{r}$ and $f_{e}$. It may happen that $f_{r}<f_{e}$, so $x_{r}$ would be a better new point than $x_{e}$, and $x_{e}$ is still accepted for the new simplex. The working simplex is kept as large as possible, to avoid premature termination of iterations, which is sometimes useful for non-smooth functions [13].

- Contract: If $f_{r} \geq f_{s}$, compute the contraction point $x_{c}$ by using the better of the two points $x_{h}$ and $x_{r}$.

○ Outside: If $f_{s} \leq f_{r}<f_{h}$, compute

$x_{c}:=c+\beta\left(x_{r}-c\right)$ and

$f_{c}:=f\left(x_{c}\right)$. If $f_{c} \leq f$, accept $x_{c}$ and terminate the iteration. Otherwise, perform a shrink transformation

○ Inside: If $f_{r} \geq f_{h}$, compute $x_{c}:=c+\beta\left(x_{h}-c\right)$ and $f_{c}:=f\left(x_{c}\right)$. If $f_{c}<f_{h}$, accept $x_{c}$ and terminate the iteration. Otherwise, perform a shrink transformation.

- $\quad$ Shrink: Compute $n$ new vertices $x_{j}:=x_{l}+\delta\left(x_{j}-x_{l}\right)$ and $f_{j}:=f\left(x_{j}\right)$, for $j=0, \ldots . ., n$, with $j \neq l$.

The shrink transformation was introduced to prevent the algorithm from failing in the following case:

A failed contraction is much rarer, but can occur when a valley is curved and one point of the simplex is much farther from the valley bottom than the others; contraction may then cause the reflected point to move away from the valley bottom instead of towards it. Further contractions are then useless. The action proposed contracts the simplex towards the lowest point, and will eventually bring all points into the valley.

2.3.3 Termination tests

A practical implementation of the Nelder-Mead method must include a test that ensures termination in a finite amount of time. The termination test is often composed of three different parts: term_x,term_f and fail.

- $\quad$ term $x$ is the domain convergence or termination test. It becomes true when the working simplex $S$ is 
sufficiently small in some sense (some or all vertices $x_{j}$ are close enough).

- $\quad t e r m \_f$ is the function-value convergence test. It becomes true when (some or all) function values $f_{j}$ are close enough in some sense.

- fail is the no-convergence test. It becomes true if the number of iterations or function evaluations exceeds some prescribed maximum allowed value.

The algorithm terminates as soon as at least one of these tests becomes true.

Various forms of term_x and term $\_$f tests have been used in practice, and some common implementations of the algorithm have only one of these two tests $[14,15]$.

If the algorithm is expected to work for discontinuous functions $\boldsymbol{f}$, then it must have some form of a term_x test. This test is also useful for continuous functions, when a reasonably accurate minimizing point is required, in addition to the minimal function value. In such cases, a term $\_$test is only a safeguard for "flat" functions.

Table 1: Values of optimizing parameters for different normalized frequency for Step index profile

\begin{tabular}{cccc}
\hline$\alpha$ & $R_{0}$ & $\mu$ & $V$ \\
\hline 1.068963 & 1.190014 & 2.033124 & 1.4000 \\
0.810013 & 0.894742 & 3.086655 & 1.6000 \\
2.139003 & 1.311694 & 4.595406 & 1.8000 \\
1.708235 & 1.086141 & 6.939758 & 2.0000 \\
1.533399 & 0.970482 & 11.088520 & 2.2000 \\
1.142917 & 0.799907 & 20.448212 & 2.4000 \\
0.033122 & 0.131163 & 61.545382 & 2.6000 \\
1.839455 & 0.941374 & 61.719697 & 2.8000 \\
0.726407 & 0.572523 & 61.543884 & 3.0000 \\
0.000570 & 0.015588 & 62.724726 & 3.2000 \\
\hline
\end{tabular}

Table 2: Values of optimizing parameters with different normalized frequency for parabolic-index profile.

\begin{tabular}{cccc}
\hline$\alpha$ & $R_{0}$ & $\mu$ & $V$ \\
\hline 1.356165 & 1.258932 & 1.314966 & 1.7500 \\
\hline 1.451176 & 1.070906 & 1.925299 & 2.0000 \\
\hline 1.245270 & 0.864681 & 2.651519 & 2.2500 \\
\hline 1.019513 & 0.705841 & 3.518595 & 2.5000 \\
\hline 0.988457 & 0.641018 & 4.557522 & 2.7500 \\
\hline 1.582103 & 0.759293 & 5.806230 & 3.0000 \\
\hline 1.050838 & 0.585569 & 7.310931 & 3.2500 \\
\hline 1.619058 & 0.693173 & 9.128715 & 3.5000 \\
\hline 0.560740 & 0.391322 & 11.329089 & 3.7500 \\
\hline 0.854820 & 0.465577 & 13.997434 & 4.0000 \\
\hline
\end{tabular}

\section{RESULTS AND DISCUSSIONS}

Detailed comparison between proposed formulation and available exact results [18] has been carried out in this section, to investigate the accuracy of the proposed approximation of fundamental modal field. It has been justified by many authors [2-6], that two parameter approximation is more accurate than single parameter approximation. The proposed approximation of fundamental field involves three optimizing parameters, which incorporates more flexibility to modify design parameters for an optical fibers having wide range of specifications. Optimized values of these parameters for different normalized frequencies are given in Table 1 and Table 2 for step and parabolic index profile respectively, so that one can use it directly for practical purposes. Values for other normalized frequencies can also be obtained easily, if required.
In order to verify the feasibility of the proposed approximation, a comparison is made between the results of proposed semi analytical formulation and the exact results [18]. Variation of Effective index (Fig. 1,2) and effective area (Fig. $3,4)$ as a function of normalized frequency, are plotted for step and parabolic index profile respectively. The curves in figures corresponding to the results obtained and exact numerical result are indistinguishable, particularly in the lower normalized frequency region, where the use of a simple Gaussian function may lead to inaccurate result.

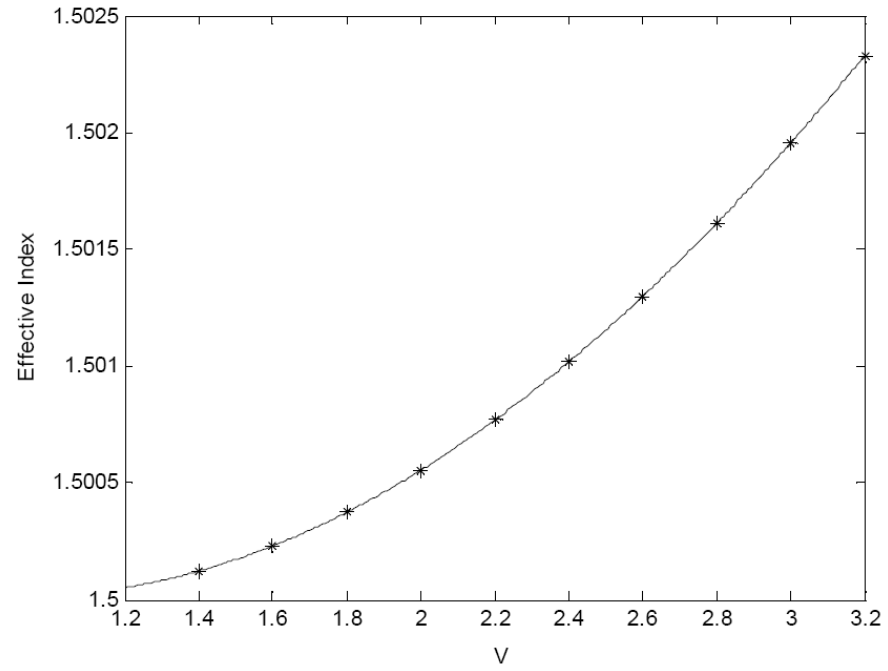

Figure.1. Variation of Effective Index with normalized frequency $(V)$ for step-index profile (- exact results; *** results by our approximation.).

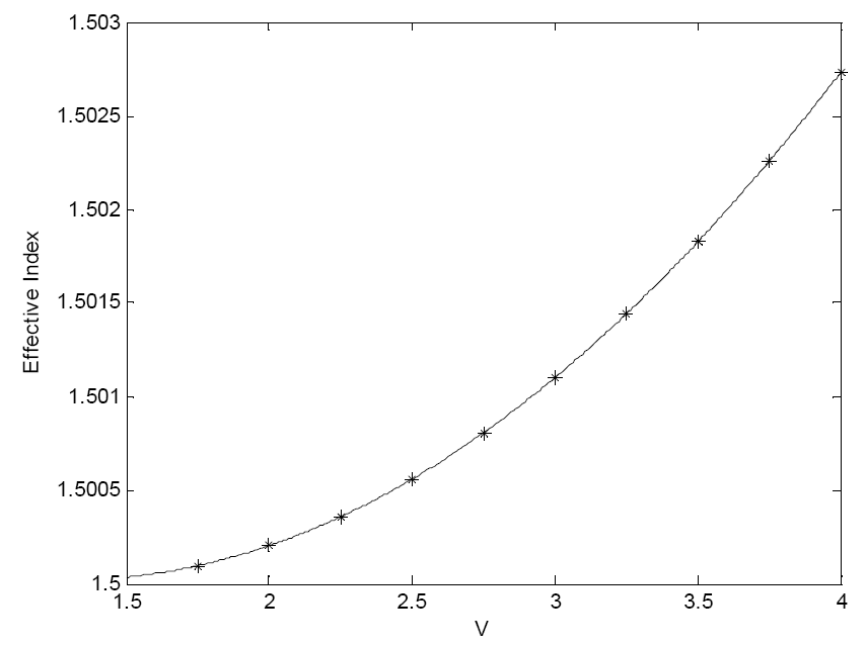

Figure.2. Variation of Effective Index with normalized frequency $(V)$ for parabolic-index profile (- exact results; *** results by our approximation.).

\section{CONCLUSIONS}

An accurate three parameter approximation of fundamental modal field for an optical fiber has been presented, which can be effectively used to estimate effective index and effective area of graded-index. Taking step and parabolic index fiber as examples, it has been shown that the results obtained with the proposed function are excellently matching with the exact results [18]. Besides providing values of optimizing parameters involved in the approximate field, all related analytical expressions have also been presented which can be used directly 
by optical fiber designer while predicting effective area of an optical fiber, for a wide range of normalized frequencies. These results can be also used as reference results to verify the accuracy of other approximate models.

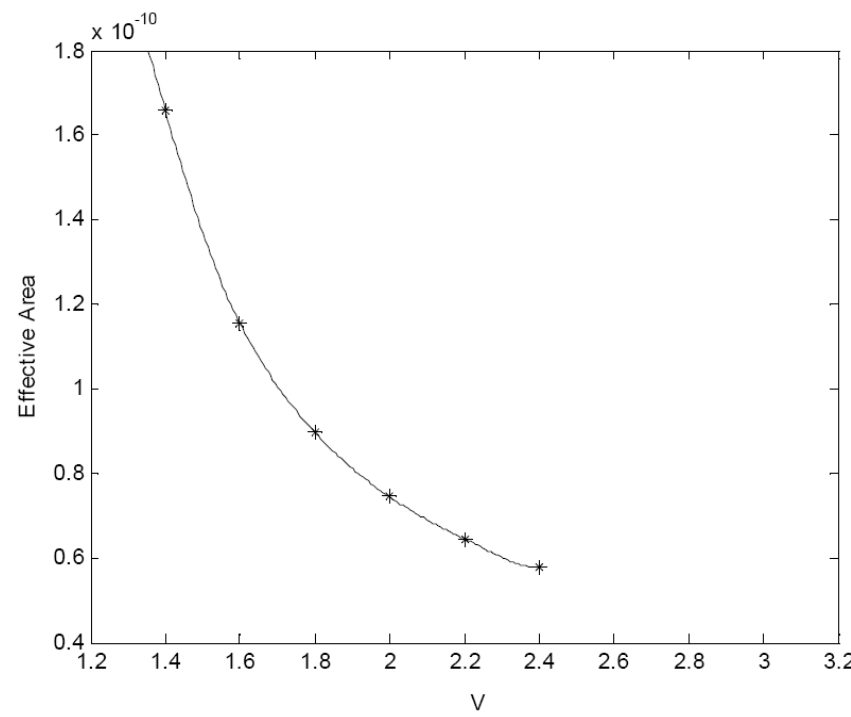

Figure.3. Variation of Effective area with normalized frequency $(V)$ for step-index profile (- exact results; *** results by our approximation.).

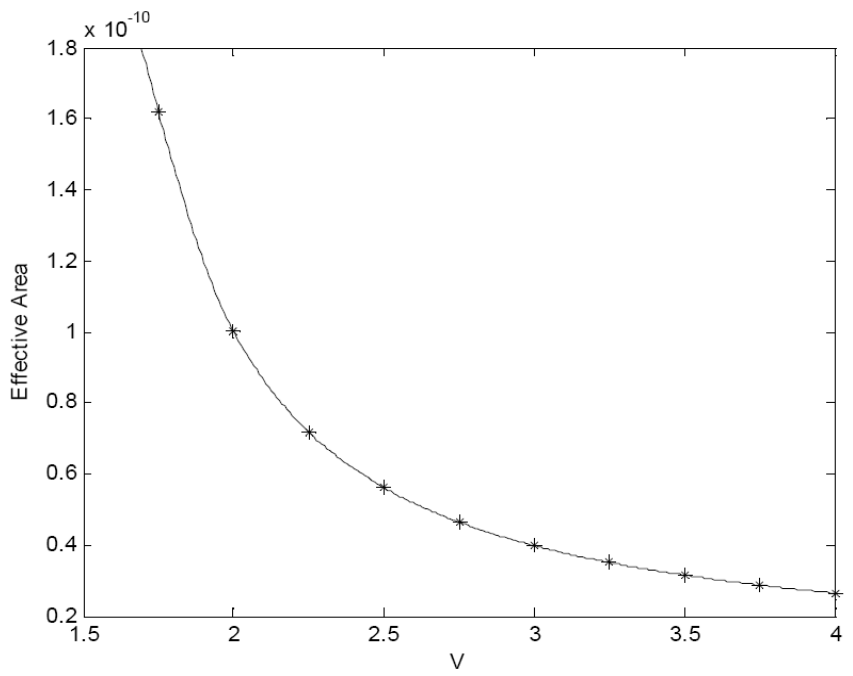

Figure.4. Variation of Effective area with normalized frequency $(V)$ for parabolic-index profile (— exact results; *** results by our approximation.).

\section{REFERENCE}

[1] D. Marcuse, "Gaussian approximation of the fundamental mode of graded-index fibers," J. Opt. Soc. Am. 68, 103-109 (1978).

[2] A. Sharma and A. K. Ghatak, "A variational analysis of single mode graded-index fibers," Opt. Comm. 36, 22-24 (1981)

[3] A. Sharma, S. I. Hossain and A. K. Ghatak, "The fundamental mode of graded-index fibers: Simple and accurate variational methods," Opt. and Quantum Elect. 14, 7-15 (1982).

[4] S. C. Chao, W. H. Tasi and M. S. Wu, "Extended
Gaussian approximation for single-mode graded-index fibers," J. Lightwave Tech. 12, 392-395 (1994).

[5] A. Ankiewicz and G. D. Peng, "Generalized Gaussian approximation for single-mode fibers" J. Lightwave Tech. 10 (1), 22-27 (1992).

[6] G. De Angelis, G. Panariello and A. Scaglione, "A variational method to approximate the field of weakly guiding optical fibers by Laguerre-Gauss/Bessel expansion," J. Lightwave Tech. 17, 2665-2674 (1999).

[7] Q. Cao and S. Chi, "Approximate analytical description for fundamental-mode fields of graded-index fibers: Beyond the Gaussian approximation," J. Lightwave Tech. 18 (1), 54-59 (2001).

[8] A. K. Ghatak, R. Srivastava, I. F. Faria, K. Tyagarajan and R. Tewari, "Accurate method for characterizing single mode fibers: Theory and Experiment," Elect.Lett. 19(3), 97-99 (1983).

[9] J.C. Lagarias, , J.A. Reeds, M.H. Wright, and P.E. Wright,., "Convergence Properties of the Nelder- Mead Simplex Method in Low Dimensions", SIAM J. Optim. 9 , pp. 112-147(1998).

[10] J.M. Parkinson and , D. Hutchinson., "An investigation into the efficiency of variants on the simplex

method", in Numerical Methods for Nonlinear Optimization, F.A. Lootsma (Ed.), (Academic Press, 1972) pp. 115-135.

[11] M.H. Wright, "Direct Search Methods: Once Scorned, Now Respectable", in Numerical Analysis

1995, Proceedings of the 1995 Dundee Biennial Conference in Numerical Analysis, D.F. Griffiths and G.A. Watson (Eds.), (Addison Wesley Longman, 1996), pp. 191-208.

[12] J.A. Nelder and R. Mead, "A simplex method for function minimization”, Comput. J., 7, pp. 308-313(1965).

[13] T.H Rowan, "Functional Stability Analysis of Numerical Algorithms", Ph.D. thesis, (University of Texas, Austin, 1990).

[14] Sanja. Singer and Saša. Singer, "Complexity Analysis of Nelder-Mead Search Iterations", in Proceedingsof the 1. Conference on Applied Mathematics and Computation, Dubrovnik, Croatia, 1999, M. Rogina, V. Hari, N. Limić, and Z. Tutek (Eds.), (PMF-Matematički odjel, Zagreb, 2001) pp. 185-196.

[15] Saša. Singer, and Sanja. Singer, , "Efficient Implementation of the Nelder-Mead Search Algorithm",Appl. Numer. Anal. Comput. Math. 1, No. 3, pp. 524-534(2004).

[16] I. S. Gradshteyn and I. M Ryzhik, Table of Integrals, series and products, (Academic, 1980).

[17] M.Abramowitz and I. A Stegun, Handbook of Mathematical Functions, (Dover, 1981).

[18] K. Kawano, T. Kitoh: "Introduction to Optical Waveguide Analysis", (John Willy and Sons, 2001), 